\title{
Enhancement of the FIDA diagnostic at ASDEX Upgrade for velocity space tomography
}

\author{
M. Weiland ${ }^{1}$, B. Geiger ${ }^{1}$, A. S. Jacobsen ${ }^{2}$, M. Reich ${ }^{1}$, M. Salewski ${ }^{2}$, T. Odstrčil ${ }^{1}$ and the \\ ASDEX Upgrade Team ${ }^{1}$ \\ ${ }^{1}$ Max-Planck-Institut für Plasmaphysik, 85748 Garching, Germany \\ ${ }^{2}$ Technical University of Denmark, Department of Physics, Dk-2800 Kgs. Lyngby, Den- \\ mark
}

Corresponding author: markus.weiland@ipp.mpg.de

\begin{abstract}
Recent upgrades to the FIDA (fast-ion D-alpha) diagnostic at ASDEX Upgrade are discussed: The diagnostic has been extended from three to five line of sight arrays with different angles to the magnetic field, and a spectrometer redesign allows the simultaneous measurement of red- and blue-shifted parts of the Doppler spectrum. These improvements make it possible to reconstruct the 2D fast-ion velocity distribution $f\left(E, v_{\|} / v\right)$ from the FIDA measurements by tomographic inversion under a wide range of plasma parameters. Two applications of the tomography are presented: A comparison between the distributions resulting from $60 \mathrm{keV}$ and 93 $\mathrm{keV}$ NBI and a velocity-space resolved study of fast-ion redistribution induced by a sawtooth crash inside and outside the sawtooth inversion radius.
\end{abstract}

\section{Introduction}

Fast, supra-thermal ions are created in present-day fusion devices by neutral beam injection (NBI) and ion cyclotron resonance heating (ICRH), and they are used for plasma heating and current drive. In burning plasmas, fast $\alpha$ ions from fusion reactions will be the main heating source. It is therefore crucial to ensure a good confinement of fast ions and understand their transport behavior.

Fast ions can be studied e.g. with neutral particle analyzers [1], collective Thomson scattering [2] or with neutron yield measurements and spectroscopy [3, 4]. In this paper, we want to focus on another possibility, which is the spectroscopic observation of line radiation (fast-ion D-alpha, FIDA) that emerges after charge exchange reactions [5]. Here, the fast ions can be distinguished from the thermal particles through the strong Doppler shift of the associated emission wavelength. Radial profiles of the FIDA emission can be measured and compared to theoretical predictions by forward-modeling with FIDASIM 6].

An analysis of the whole Doppler spectrum yields information about the 2D velocity distribution $f\left(E, v_{\|} / v\right)$, where $E$ is the energy and $v_{\|} / v$ is the pitch of the fast ions. The observed Doppler-shift of an ion is proportional to the projection of its velocity vector (after neutralization) onto the line of sight direction. Therefore, the FIDA spectrum of 
a LOS with angle $\Phi$ to the magnetic field is dominated by fast-ions with similar pitch angles (i.e. $\left.\cos \left(v_{\|} / v\right) \approx \Phi\right)$. Observation from different viewing angles allows consequently a tomographic reconstruction of $f\left(E, v_{\|} / v\right)$ [7]. This has been demonstrated successfully at ASDEX Upgrade from three FIDA viewing angles [8] in a very thin plasma, which allowed to measure the full D-alpha spectrum without blocking the bright, central D-alpha line from the plasma edge.

In the present paper, we discuss the diagnostic upgrades for the 2014 campaign, which allow to measure the full $\mathrm{D}_{\alpha}$ spectrum (i.e. blue and red Doppler shifts) permanently and under all plasma conditions. Furthermore, the FIDA diagnostic has been extended from three to five viewing arrays for a better coverage of the velocity space. First results from the new spectrometer setup and four FIDA views have been shown already in [9]. The details of the diagnostic improvements are now described in this paper in section 2 . In section 3, a first tomographic reconstruction of $f\left(E, v_{\|} / v\right)$ from all five views is presented in the presence of $60 \mathrm{keV}$ and $93 \mathrm{keV} \mathrm{NBI}$. In section 4, the fast-ion redistribution in the velocity space induced by a sawtooth crash is studied using all five FIDA views at two positions, in- and outside of the sawtooth inversion.

\section{Diagnostic setup}

\subsection{Spectrometer}

The FIDA diagnostic is based on the spectroscopic analysis of the D-alpha Doppler spectrum. The unshifted D-alpha line is typically very intense and cannot be measured simultaneously with the much weaker FIDA emission due to the limited dynamical range (16 bit) of the CCD-Camera. Therefore, it needs to be blocked. In the previous spectrometer setup (as described in [10]), this was achieved with a high pass interference filter. The drawback of this method is that only one half of the FIDA Doppler-spectrum can be measured (in that case the red-shifted part). In order to gain information about the full fast-ion velocity space, it is desirable to measure the full Doppler spectrum. Therefore, a sharp notch filter with a width of approximately 1-2 $\mathrm{nm}$ is needed. Since optical filters with these capabilities are rare and expensive, the D-alpha line is instead filtered geometrically with a wire, as it was already succesfully done e.g. in [11, 12]. Therefore, an additional lens is needed to create a sharp image on the wire. The complete setup is shown in fig. 1. The light is transferred from the plasma into the spectrometer by a vertically stacked array of glass fibers. It is then made parallel by the first lens and gets reflected and dispersed by the grating. The second lens focuses the light onto the wire, whose position has to be adjusted such that it blocks the central D-alpha line. Then, the light is focused onto the CCD-chip with the third lens.

The wavelength calibration of the spectrometer is done by measuring the known spectrum of a neon lamp on each spectrometer channel (before and after an experimental campaign). Furthermore, the same neon lamp spectrum is measured on the first spectrometer channel after each tokamak discharge to ensure that the wavelength calibration is valid for every discharge. The neon lamp is located in the spectroscopy laboratory (i.e. close to the spectrometer), and it is assumed that the wavelength does not change on the further optical path from the plasma. 


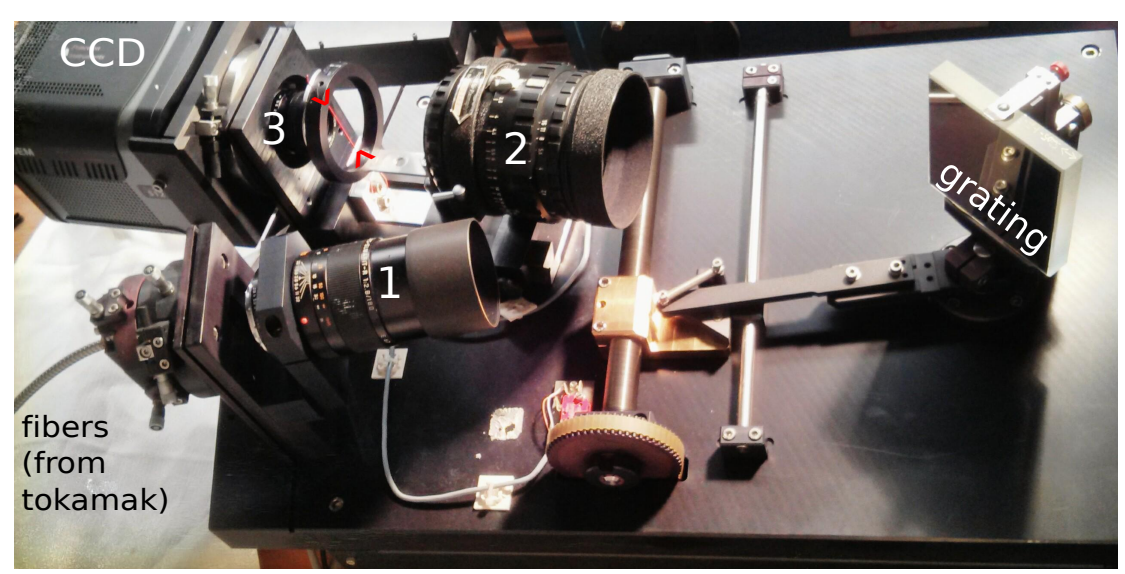

Figure 1: The FIDA spectrometer at ASDEX Upgrade. The grating has 2000 lines $/ \mathrm{mm}$ and the focal lengths of the three lenses are: $f_{1}=180 \mathrm{~mm}, f_{2}=150 \mathrm{~mm}$ and $f_{3}=25 \mathrm{~mm}$. The wire (to block the $\mathrm{D}_{\alpha}$-line) is marked with red arrows and has a width of $0.8 \mathrm{~mm}$.

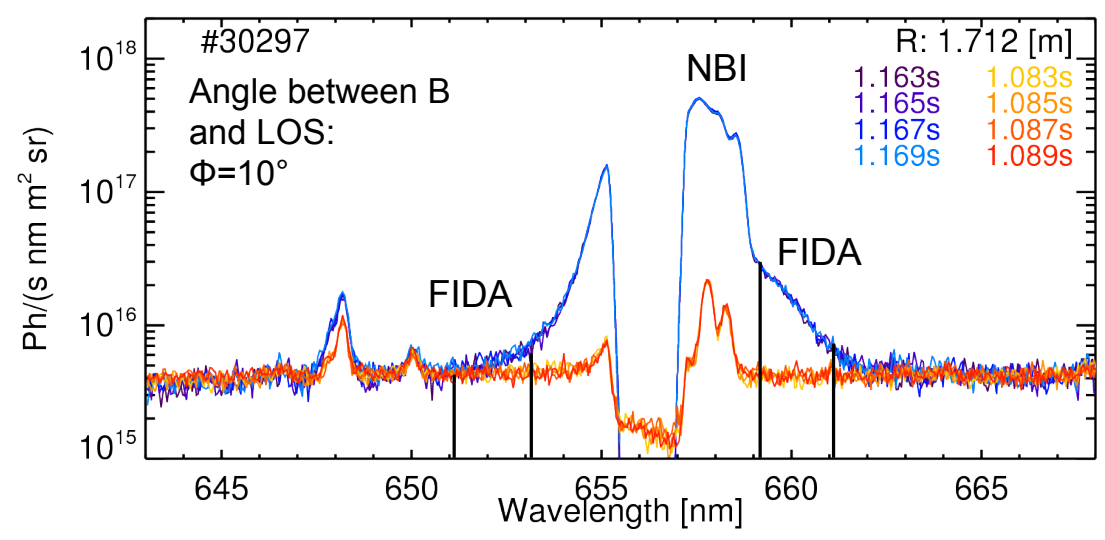

Figure 2: D-alpha spectrum measured with the new spectrometer setup. For the wavelengths marked with vertical lines, the corresponding weight functions are shown in fig. 3. The red curves correspond to the passive background radiation, measured during a NBI-off phase.

The spectrometer setup as described in [10] has been capable of measuring 16 LOS channels. Within the spectrometer upgrade, this has been extended to 27 channels, which can be measured with a time resolution of $2.5 \mathrm{~ms}$. This increase has been achieved, because the modified optics have been chosen such that the image projected onto the CCD is smaller. Consequently, the observable wavelength range has increased by a similar factor $(\approx 25 \mathrm{~nm}$ instead of $\approx 14 \mathrm{~nm}$ ), which is beneficial for measuring both the blue and red side of the Doppler spectrum. In addition, a second spectrometer with a similar design and 10 channels (20 ms time resolution) has been brought into operation permanently for FIDA measurements, which gives us the possibility to routinely measure 37 lines of sight simultaneously.

A spectrum measured with the upgraded spectrometer is shown in fig. 2, The wire-filter is clearly seen, and covers the central D-alpha line entirely, as desired. The beam emission 
is still visible, and FIDA light can now be observed on both sides of the spectrum. The spectral resolution is $0.21 \mathrm{~nm}$. For comparison, a background spectrum, measured during an NBI-off phase, is shown with the red lines. It is shifted to similar background (evaluated around $665 \mathrm{~nm}$ ) to allow a shape comparison. It can be seen that the background is mainly a flat line, which can be identified with bremsstrahlung, with some additional impurity lines. The FIDA contribution can hence be well recognized by its shape.

\subsection{Interpretation of the spectrum with weight functions}

The Doppler-spectrum contains information about the fast-ion velocity distribution. This can be analyzed quantitatively with weight functions $W$ : Each wavelength bin $\lambda$ of the FIDA spectrum can be interpreted as an integral over $f(E, \xi)$, weighted with $W$ :

$$
F I D A(\lambda)=\int_{0}^{\infty} \int_{-1}^{+1} W(\lambda, E, \xi) f(E, \xi) \mathrm{d} E \mathrm{~d} \xi
$$

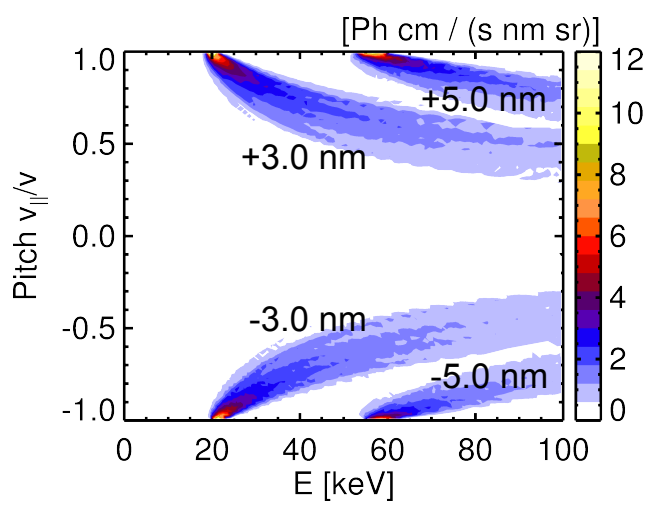

Figure 3: Weight functions for the $\Phi \approx 10^{\circ}$ view (comp. fig. 2), for four different Dopplershifts $\Delta \lambda$ with respect to the D-alpha line $\lambda_{0}=656.1 \mathrm{~nm}$.

Hereby, the pitch is denoted with $\xi:=v_{\|} / v . W$ can be calculated with the FIDASIM code [6, 13]. In fig. 3, weight functions for one LOS and four different wavelength bins are shown as an example. It can be seen that all weight functions have similar shape but cover different energy ranges. Higher Doppler-shifts $\Delta \lambda$ are connected with a weight function at higher energies, and different signs of the Doppler-shift correspond to opposite parts in the velocity space with respect to the pitch $v_{\|} / v$. The weight functions belonging to $\pm 3 \mathrm{~nm}$ (w.r.t. the unshifted D-alpha line $\lambda_{0}=656.1 \mathrm{~nm}$ ) typically mark the lower boundary of the FIDA spectrum. At lower Doppler-shifts, other contributions, e.g. from the NBI or the thermal halo neutrals become important or dominate the spectrum. Consequently, we can only measure the fast-ion velocity space above roughly $20 \mathrm{keV}$.

The weight functions can be interpreted as lines of sight (or areas of sight) in the velocity space. If enough weight functions are available, a tomographic reconstruction becomes feasible. If the spectrum in fig. 2 is binned to $0.1 \mathrm{~nm}$ wavelength bins, and if we study Doppler-shifts in a typical range of $\Delta \lambda \in \pm[3,8] \mathrm{nm}$ we get already $\approx 100$ measurements and weight functions. However, they have all similar shape and do not cover the entire 
velocity space (especially the area around pitch 0 is not covered). Therefore, additional FIDA views with different projection angles $\Phi$ (i.e. the angle between the LOS and the B-field) are needed.

\subsection{Lines of sight}

As described in [10], the FIDA diagnostic at ASDEX Upgrade had already line of sight arrays from three viewing directions: $\Phi \approx 10^{\circ},-20^{\circ}, 70^{\circ}$ (all aligned on NBI beam Q3). The minus symbol indicates that the observation is done from the opposite direction with respect to the magnetic field, which means that the Doppler spectrum is mirrored with respect to the D-alpha line. Since we are able to measure both sides of the Doppler spectrum, the sign of $\Phi$ does not matter for the information, which we get about the velocity space. This line of sight setup has now been extended to five views. The new lines of sight are chosen such that the measurements complement the three existing views in the best possible manner. To determine the optimum new angles $\Phi_{1}$ and $\Phi_{2}$ between the lines of sight and the magnetic field, we calculate synthetic spectra for the three existing views and two new views at observation angles $\Phi_{1}$ and $\Phi_{2}$ for the fast-ion velocity distribution function of beam Q3. We then calculate tomographic inversions of the synthetic spectra with added noise for the possible combinations of the angles $\Phi_{1}$ and $\Phi_{2}$ and compare the inversion with the original function by a figure of merit $Q=\left|f-f_{\text {tomo }}\right|^{2} /|f|^{2}$, where $|\cdot|$ represents the two-norm of a vector holding the function values of $f$. Optimum viewing angles for the two new views are the global minima of this figure of merit in fig. 4 at about 85 and 50 degrees. Local maxima correspond to a poor choice of the new geometry and occur when $\Phi_{1}$ or $\Phi_{2}$ are similar to one of the existing viewing angles or similar to each other. We note that the graph is symmetric about the angle bisector $\Phi_{1}=\Phi_{2}$ as $\Phi_{1}$ and $\Phi_{2}$ are interchangeable.

Hence, two new viewing arrays with $\Phi \approx-50^{\circ}$ and $\Phi \approx-85^{\circ}$ have been installed into ASDEX Upgrade (see fig. 5). The $\Phi \approx-50^{\circ}$ view is equipped with seven radial lines of sight, and the $\Phi \approx-85^{\circ}$ has five radial lines of sight. The third (middle) LOS of $\Phi \approx-85^{\circ}$ has a bad fiber with low transmission, and is not shown in the figure. The exact projection angle changes slightly over the different radial LOS of one view. Figure 6 shows an overview of the projection angles of all five views as a function of the major radius $R$. It can be seen that the angle of one LOS array varies along the plasma radius due to the geometrical arrangement of the viewing chords. It is therefore not possible to achieve an optimal $\Phi$ distribution over the whole plasma radius. We have optimized the LOS angles in the plasma center, and the $\Phi$ angles mentioned above correspond to the values in the plasma center.

In the default setup, the LOS of the $\Phi=-20^{\circ}$ view are connected to the spectrometer with $20 \mathrm{~ms}$ time resolution, while the other four viewing arrays are connected to the spectrometer with the faster CCD-camera $(2.5 \mathrm{~ms})$. If data analysis using all five views is desired, the data from the faster CCD-camera is typically averaged over $20 \mathrm{~ms}$ to reduce noise and to have a comparable time range for all measured spectra. 


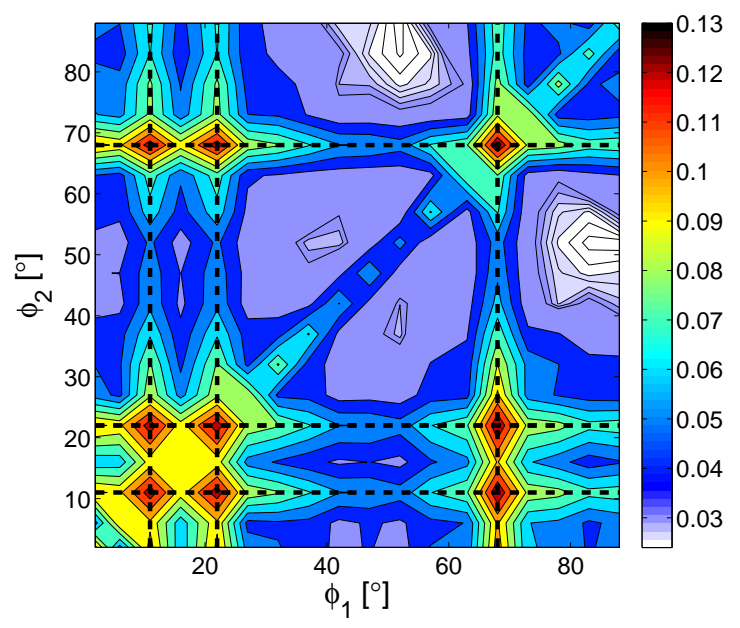

Figure 4: Figure of merit $Q=\left|f-f_{\text {tomo }}\right|^{2} /|f|^{2}$ between original fast-ion distribution $f$ and calculated tomography $f_{\text {tomo. }}$. The tomography has been calculated from five FIDA views: The three existing ones $\left(\Phi \approx 10^{\circ},-20^{\circ}, 70^{\circ}\right)$ and two new views $\Phi_{1}$ and $\Phi_{2} . \Phi_{1}$ and $\Phi_{2}$ have been varied to determine the optimal angles for the new views. The angles of the three existing views are marked with dashed lines. The global minimum and hence the optimal angles are found for $\Phi_{1,2} \approx 50^{\circ}, 85^{\circ}$. A local minimum is found for $\Phi_{1,2} \approx 40^{\circ}, 52^{\circ}$, but it is clearly less optimal than the global minimum.

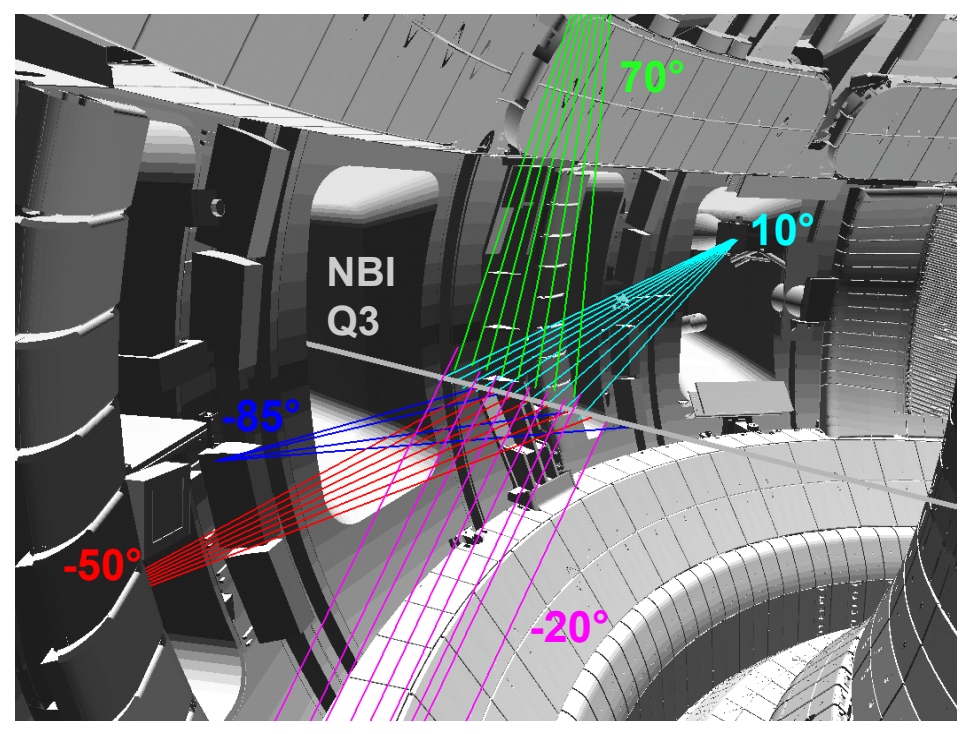

Figure 5: 3D visualization of the five FIDA line of sight arrays, along with the approximate angle $\Phi$ between the most central line of sight of each array and the local magnetic field. Negative angles indicate that the observation is from the opposite side, and hence the Doppler spectra are mirrored. It can be seen that all lines of sight are well aligned along NBI source Q3. 


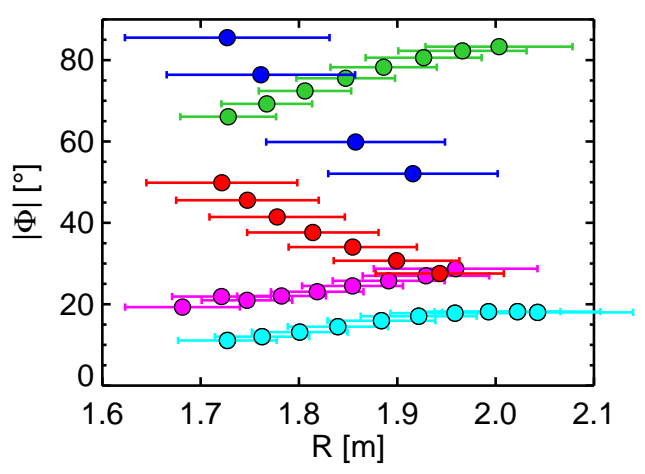

(a)

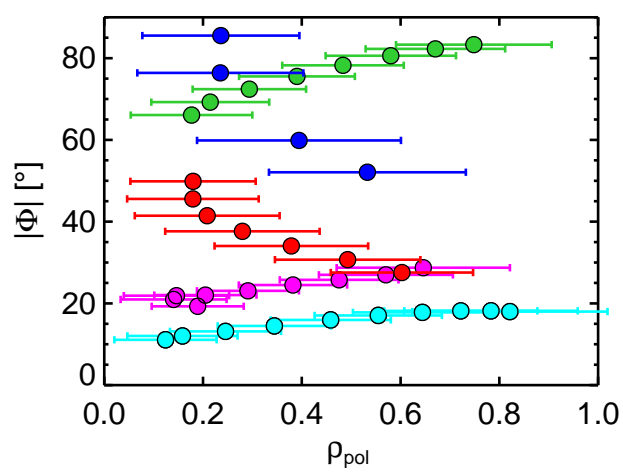

(b)

Figure 6: Absolute value of the angle between the line of sight and the magnetic field $|\Phi|$, for all lines of sight, plotted as a function of (a) major radius $R$ and (b) normalized poloidal flux radius $\rho_{\mathrm{pol}}$. The error bars correspond to the radial resolution (see section 2.4). The five viewing arrays are marked with different colors (corresponding to fig. 5). The magnetic axis is located at $R=1.72 \mathrm{~m}$.

\subsection{Radial resolution}

The radial resolution of a line of sight is mainly determined by the path of the LOS through the NBI beam. The width of the LOS can hereby be neglected in good approximation, and the width of the NBI beam and the surrounding neutral halo is the important quantity. Good radial resolution (in terms of $\rho_{\text {pol }}$ ) can be achieved if the LOS goes tangential to flux surfaces through the beam.

With the FIDASIM code, the radial resolution can be computed accurately. In particular, the origin of fast ions, which experience a charge-exchange reaction and contribute to the calculated FIDA signal can be calculated and is shown in fig. 7. The calculation has been done for typical ASDEX Upgrade discharge parameters and a fast-ion distribution calculated by TRANSP/NUBEAM [14, 15] resulting from $2.5 \mathrm{MW}$ of $60 \mathrm{keV}$ NBI from source Q3. For comparison, the neutral density of NBI and halo is plotted with contour lines. It can be seen that the FIDA measurement volume is localized where the LOS crosses the neutral cloud. However, there is also a significant broadening of the contribution perpendicular to the LOS, which can be explained by finite Larmor radii of the fast ions and finite free mean paths of the fast neutrals.

From these $R, z$ contribution matrices, we can compute a $R$ and $\rho$ histogram. We define our measurement position with its mean-value, and the width of the measurement with the standard deviation of the histogram. Both are shown in figure 6, together with the angle of the line of sight towards B. We can see that the $\Phi=10^{\circ}$ and $\Phi=20^{\circ}$ views have the best resolution both in $R$ and $\rho$ because they are very tangential to the flux surfaces. $\Phi=70^{\circ}$ has a comparably good $R$ resolution, because its line of sights are vertical. However, the $\rho$-resolution is worse, especially in the plasma center, because the neutral beam covers a broad $\rho$-range there. $\Phi=50^{\circ}$ is less tangential as $\Phi=10^{\circ}$ and $20^{\circ}$ and has a $\rho$ resolution comparable to the vertical view. $\Phi=85^{\circ}$ has the broadest measurement 

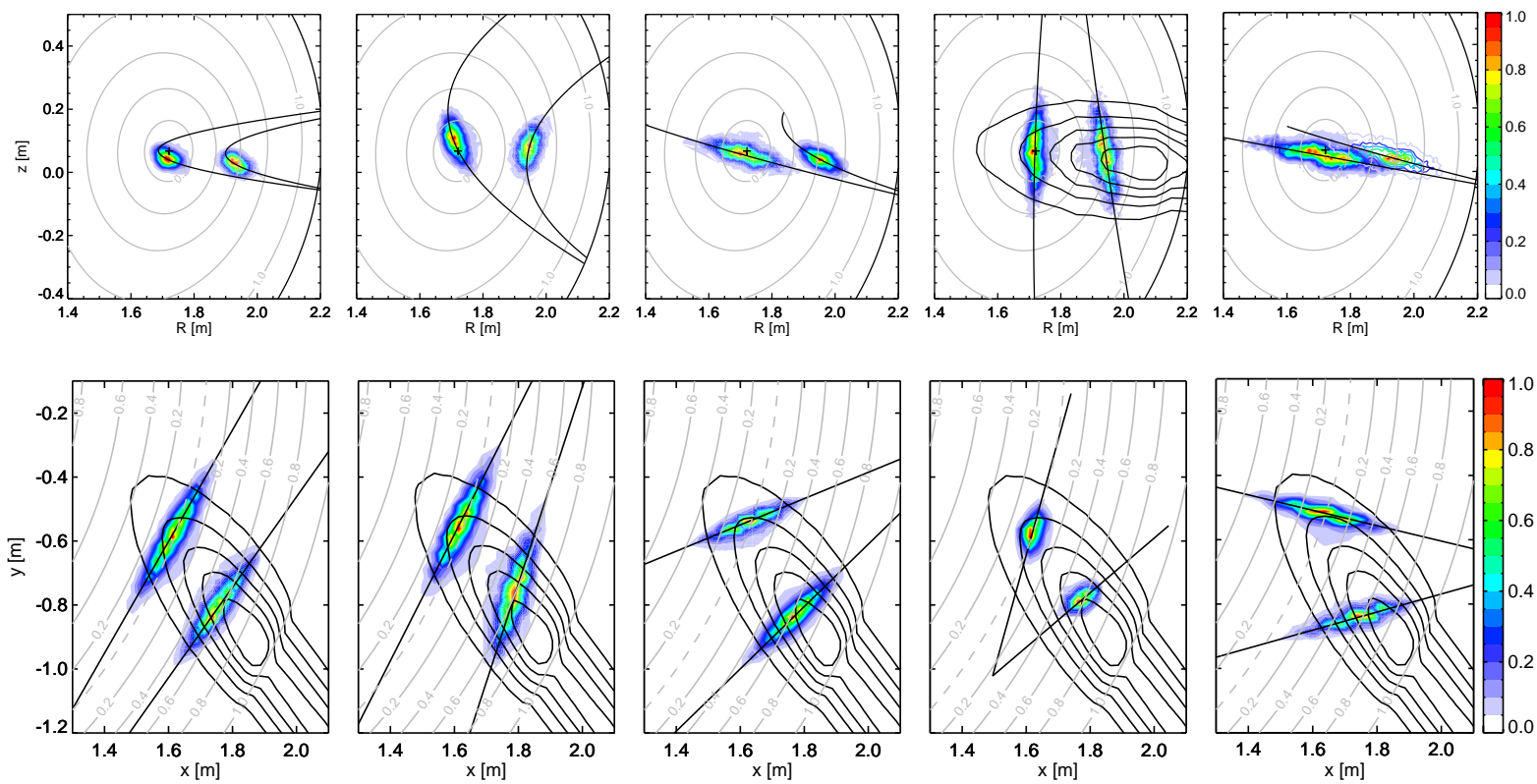

(a) $\Phi \approx 10^{\circ}$

(b) $\Phi \approx-20^{\circ}$

(c) $\Phi \approx-50^{\circ}$

(d) $\Phi \approx 70^{\circ}$

(e) $\Phi \approx-85^{\circ}$

Figure 7: The origin of fast ions, which do a charge-exchange reaction and contribute to the calculated FIDA signal is shown as colored contour on a normalized scale. This can be interpreted as the radial resolution of the FIDA diagnostic. Top: poloidal cross-section, bottom: Top-down view. The geometrical lines of sight are drawn with a continuous line, and normalized poloidal flux radius is shown in gray contours. For comparison, the sum over all neutral densities (i.e. beam + halo neutrals and all atomic states) are drawn with black contours in some of the plots.

volume, because it is least tangential to the flux surfaces, and has consequently only five radial lines of sight.

\subsection{Weight functions of the five FIDA views}

The weight functions of all five FIDA views are shown in fig. 8 as an example for a positive and a negative Doppler-shift. It can be seen that the $10^{\circ}$ view observe ions with high pitches, and the weight functions have a rather narrow shape. The other extreme case is the $85^{\circ}$ view, which dominantly observes strongly gyrating fast ions with pitches in a rather broad range around zero. It can be seen that its weight functions are much broader due to the strong influence of the gyromotion on the projected velocity. For the views in between, the shape of the weight functions changes smoothly according to the absolute value of $\Phi$. The weight functions at negative Doppler-shifts are approximately mirror-symmetric with respect to $v_{\perp} / v$ if compared to the ones at positive Doppler-shifts. In particular, Doppler-shifts $\Delta \lambda$ with $\Delta \lambda \cdot \operatorname{sgn} \Phi>0$ correspond to FIDA light from co-current fast ions, while $\Delta \lambda \cdot \operatorname{sgn} \Phi<0$ corresponds to counter-current fast ions. 


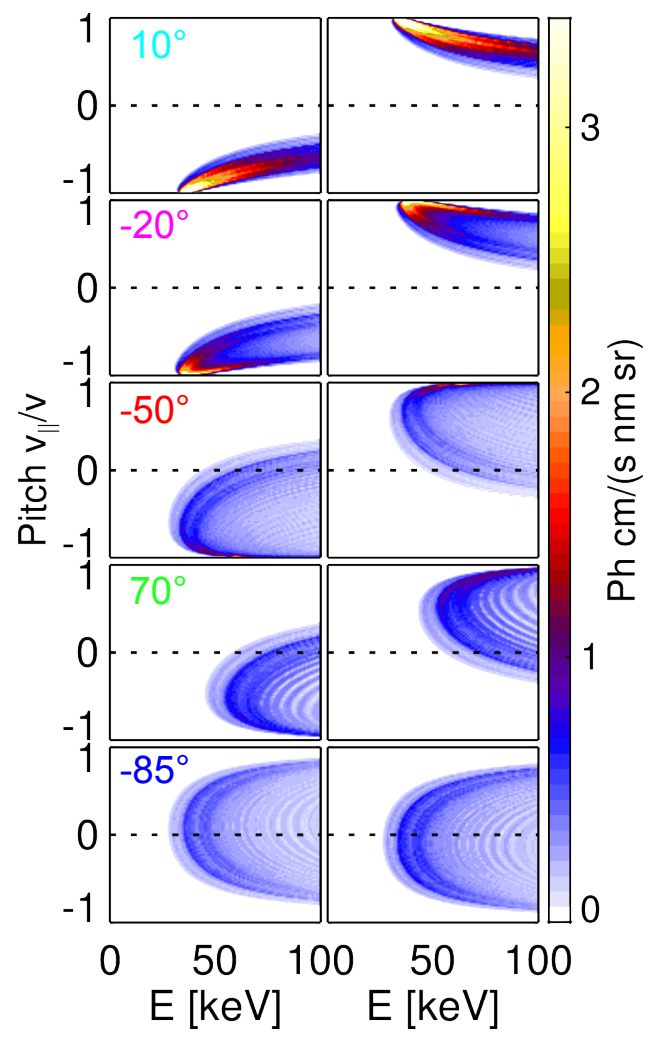

Figure 8: Weight functions of the five FIDA views and two Doppler-shifts with $\Delta \lambda=$ $\pm 3.9 \mathrm{~nm}$. The plots are aligned such that the left column fulfills $\Delta \lambda \cdot \operatorname{sgn} \Phi<0$, and the right column fulfills $\Delta \lambda \cdot \operatorname{sgn} \Phi>0$.

\subsection{Tomographic reconstruction}

In order to calculate a tomographic reconstruction of the velocity distribution $f(E, \xi)$, we need to invert eq. 1. Therefore, we discretize the equation and stack all measurements in a vector $\vec{s}$, and all pixels of the $2 \mathrm{D}$ distribution function $f(E, \xi)$ in a vector $\vec{f}$. They are then connected by a matrix $\mathrm{W}$, which contains the discretized weight functions:

$$
\vec{s}=W \vec{f}
$$

$\vec{s}$ and $\mathrm{W}$ are normalized with respect to the noise level $\sigma$ of the measurement, such that parts of the spectra with good signal-to-noise ratio are considered more strongly. The task is now to calculate the pseudo-inverse $W^{+}$of $W$, which can be done e.g. by singular value decomposition as shown in [7, 8] or by maximum entropy regularization [16]. A systematic comparison between different inversion methods can be found in [17]. Here, we use a first-order linear regularization, as described in [18]:

$$
\begin{aligned}
& W^{+}=\left(W^{\mathrm{T}} W+\alpha H\right)^{-1} W^{\mathrm{T}} \\
& H=\beta \nabla_{E}^{T} \cdot \nabla_{E}+\nabla_{\xi}^{T} \cdot \nabla_{\xi}
\end{aligned}
$$


With $\nabla_{E}$ and $\nabla_{\xi}$ we denote matrix representations of the partial derivative in $E$ and $\xi$ direction. To ensure dimensional consistence, the prefactor $\beta$ is needed. This approach is equivalent to calculating a least-squares fit, with the additional condition that the solution should be a smooth function, i.e:

$$
\text { minimize } \frac{1}{2}\|W \vec{s}-\vec{f}\|^{2}+\alpha \cdot \vec{f}^{\mathrm{T}} H \vec{f}
$$

The ratio between both conditions is given by the parameter $\alpha$. $\beta$ allows furthermore to adjust the ratio of smoothing in $E$ and $\xi$ direction. We find the tomographic reconstruction $\vec{f}$ then by the matrix multiplication:

$$
\vec{f}=W^{+} \vec{s}
$$

The so calculated tomography $\vec{f}$ can contain negative values. To overcome this nonphysical behavior, we have implemented a simple yet effective iterative method: After the first step, the most negative pixels of $\vec{f}$ are determined. For each these pixels (index $j$ ), an artificial weight-signal pair is added (i.e. a new row to $W$ and $\vec{s}$ with index $i$ ) fulfilling:

$$
W_{i, j}=W_{0} \text {, zero elsewhere, and } s_{i}=0 .
$$

This means that an artificial weight matrix is introduced, which sees just one pixel, and the corresponding signal is set to zero. $W_{0}$ is a positive number and should be large with respect to the real weight functions. This forces the selected pixel (which was negative before) to zero. This procedure can be repeated iteratively, until all negative values are eliminated or the most negative value reaches a reasonable threshold close to zero.

\section{Fast-ion velocity distribution from 60 and $93 \mathrm{keV} \mathrm{NBI}$}

In the following section, we investigate the velocity-space accuracy of the tomographic reconstruction by comparing a phase with only $60 \mathrm{keV}$ NBI and a phase with $93 \mathrm{keV}$ beams in addition. We have chosen discharge \#30950, which was run with a magnetic field of $-2.2 \mathrm{~T}$ and a plasma current of $1 \mathrm{MA}$. It features rather low central electron densities between $1.0-3.0 \cdot 10^{19} \mathrm{~m}^{-3}$, which leads to low bremsstrahlung and hence a good signal-to-noise ratio of the FIDA signal. The basic time traces are shown in fig. 9 .

We have analyzed two time points: $0.92 \mathrm{~s}$ with only $60 \mathrm{keV}$ NBI (from Q3), and 1.23s with $93 \mathrm{keV}$ from Q6 and Q8 in addition. In between, there is a short time phase without any beam, which is used to substract passive FIDA radiation. In fig. 10, the FIDA spectra are shown for the two time points for five FIDA views, which measure all approximately at the same radial position in the plasma center $\left(R=1.74 \mathrm{~m}, z=0.07 \mathrm{~m}\right.$ and $\rho_{\text {pol }}=$ $0.16,0.10$ for the first/second time point). It can be seen directly from the spectra that the FIDA radiation increases strongly. This can be interpreted with an increase of the fast-ion density - however a quantitative statement cannot be given, because the beam attenuation has also changed due to different plasma parameters in the two time points. With the FIDA tomography, quantitative statements are possible, because this effect is 


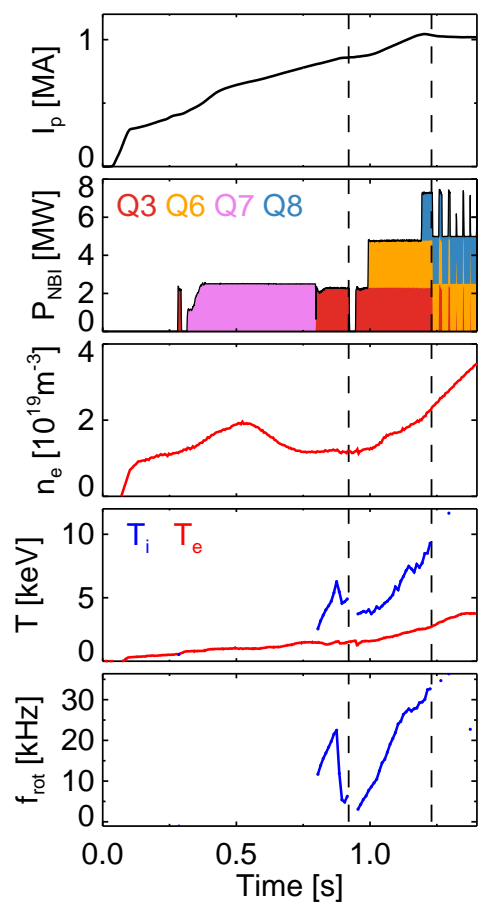

Figure 9: Relevant time traces of ASDEX Upgrade discharge \#30950. From top to bottom: Plasma current, NBI power, electron density, electron and ion temperature, toroidal plasma rotation frequency. The time traces of the last three correspond to the radial position, where we calculate the FIDA tomography. The two time points, which we have analyzed are marked with vertical dashed lines. Q3 etc. denote the different NBI sources: Q3 serves as diagnostic beam for FIDA and CXRS measurements and is operated with $60 \mathrm{keV}$ injection energy, Q6-8 are operated with $93 \mathrm{keV}$. All beams inject in the co-current direction. Q3 and Q8 are aligned on-axis with similar angle to the magnetic field, while Q6-7 are off-axis and more tangential, and hence contribute only weakly to the studied FIDA measurement position in the plasma center.

taken into account by calculating separate weight functions for each time point. The presence of $93 \mathrm{keV}$ fast ions should lead to larger Doppler shifts in the spectrum. This effect is visible in some of the spectra, but it is weak, because the $93 \mathrm{keV}$ beams inject less particles than the $60 \mathrm{keV}$ beams (at same power) and the charge exchange cross-sections go down towards higher energies.

We have performed tomographic reconstructions from these spectra. Regions with impurity lines and beam emission are excluded, as-well as the wavelength range [-3.1 nm, $+3.1 \mathrm{~nm}]$ around the D-alpha line, which is dominated by thermal ions, emission from the beam neutrals and passive radiation from the cold plasma edge. In doing so, we make sure that we get mainly information about the fast ions from the spectra. However, this also means that we do not get detailed information about fast ions below $\approx 20 \mathrm{keV}$. This limit seems to be acceptable, since still a broad region of the energy range is covered $(2 / 3$ in the case of $60 \mathrm{keV} \mathrm{NBI}$ and even more for $93 \mathrm{keV} \mathrm{NBI}$ ). In addition, the thermal ions are well-diagnosed by conventional charge-exchange diagnostics. 

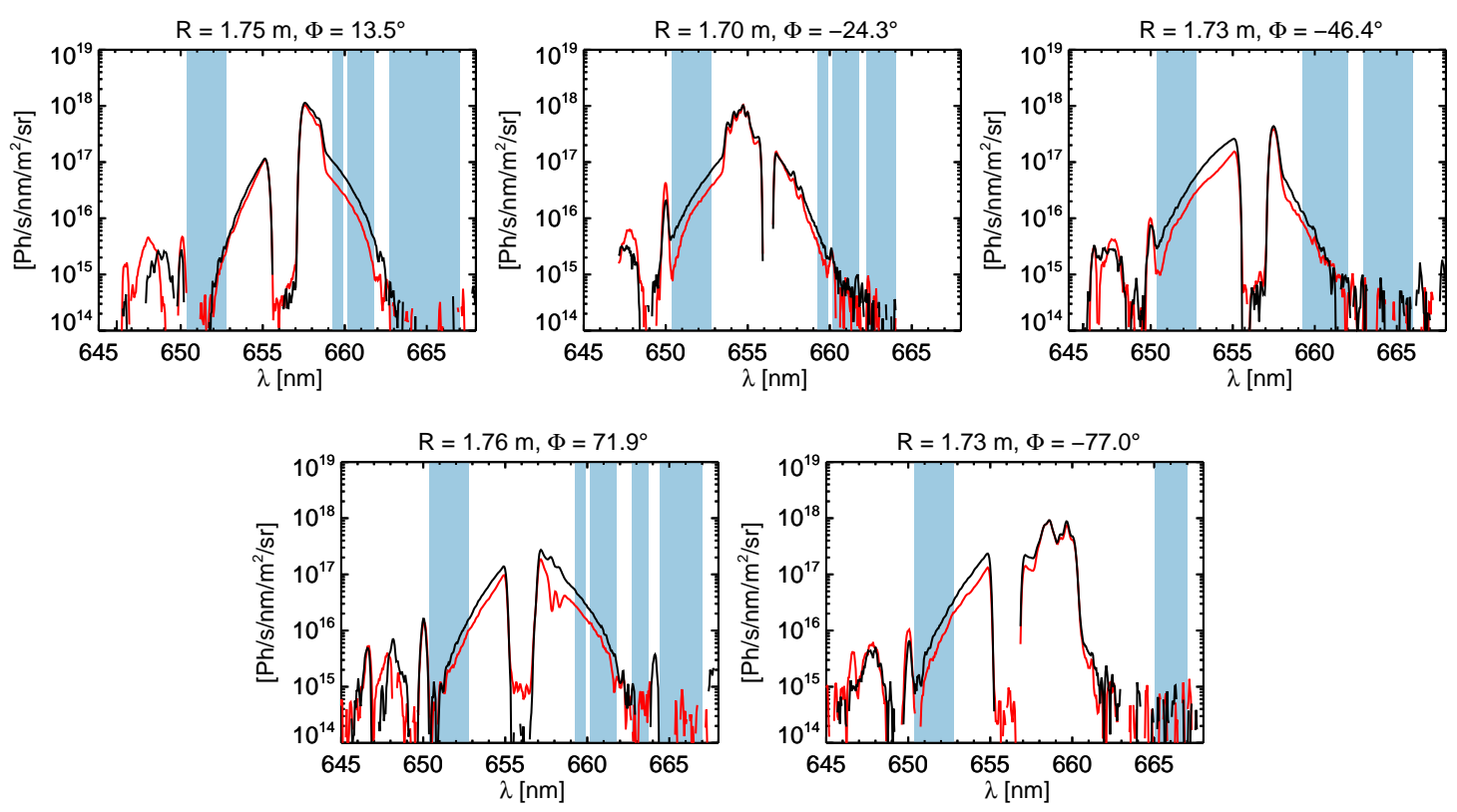

Figure 10: FIDA spectra of five views at $t=0.92 \mathrm{~s}$ ( $60 \mathrm{keV} \mathrm{NBI}$ only, red line) and $t=1.23 \mathrm{~s}$ (60 and $93 \mathrm{keV}$ NBI, black line). Passive radiation (measured during a beam-off phase) has been subtracted - therefore, the baselines of the spectra appear very noisy in the logarithmic plot, as it is close to zero. The tomography is calculated from the blue-shaded regions.

The result of the tomography is seen in fig. 11. For $t=0.92 s$, the tomography yields a velocity distribution which has almost no fast ions above $60 \mathrm{keV}$. The pitch distribution has a peak at $\approx 0.6$, which is perfectly within expectations from the beam geometry. For the second time point, $t=1.23 \mathrm{~s}$, a clear contribution from fast ions above $60 \mathrm{keV}$ is found. Moreover the fast-ion density increases strongly, which can be expected, because the NBI power is tripled. The tomography is calculated down to energies of $15 \mathrm{keV}$. It has to be noted that the region between 15 and $24 \mathrm{keV}$ is more weakly covered by weight functions than the higher energy ranges, and the results within this region should be treated with care. This is especially true for $15-20 \mathrm{keV}$, which is only (partially) covered by some of the perpendicular views, and hence has an extrapolative character.

In fig. 12, the fast-ion distribution, calculated by the NUBEAM module in TRANSP [14, 15], is shown. To ensure a realistic comparison with the FIDA tomography, a shifted Maxwellian distribution has been added to account for thermal deuterium (with density $n_{\mathrm{D}}$, temperature $T_{\mathrm{i}}$ and toroidal rotation $\left.v_{\text {rot }}\right)$ :

$$
f(E, \xi)=\frac{n_{\mathrm{D}}}{T_{\mathrm{i}}^{3 / 2}} \sqrt{\frac{E}{\pi}} \cdot \exp \left(-m \frac{\left(v_{\|}-v_{\mathrm{rot}}\right)^{2}+v_{\perp}^{2}}{2 T_{\mathrm{i}}}\right)
$$




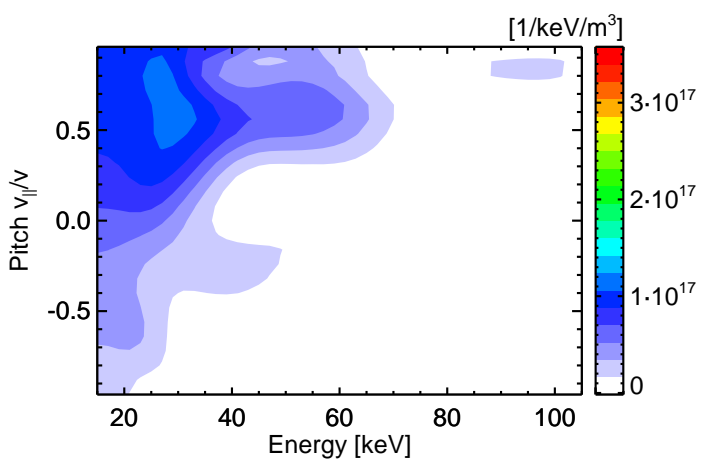

(a) $t=0.92 \mathrm{~s}$

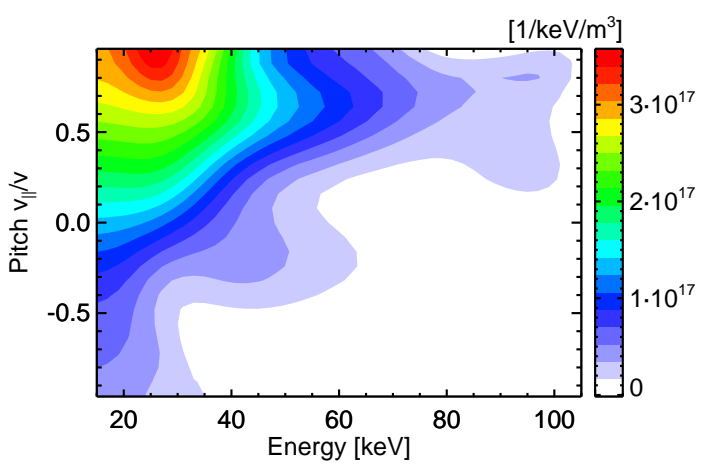

(b) $t=1.23 \mathrm{~s}$

Figure 11: Tomographic reconstruction of the ion velocity distribution function. The tomography is calculated on a 46x25 grid and has hence 1150 pixels, and 280 measurements (e.g. FIDA wavelength bins) are used.

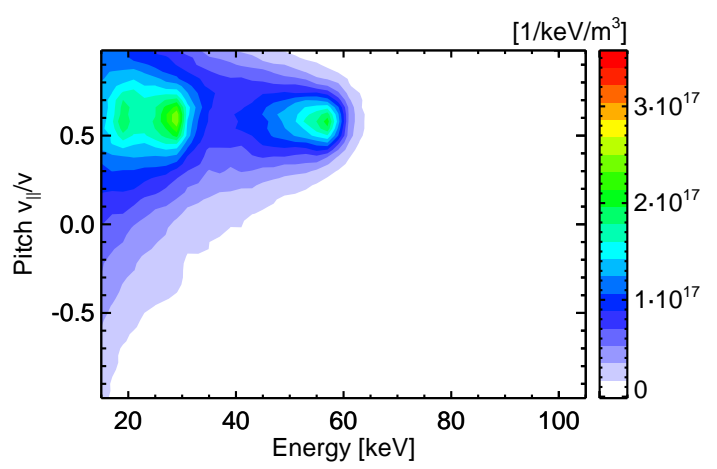

(a) $t=0.92 \mathrm{~s}$

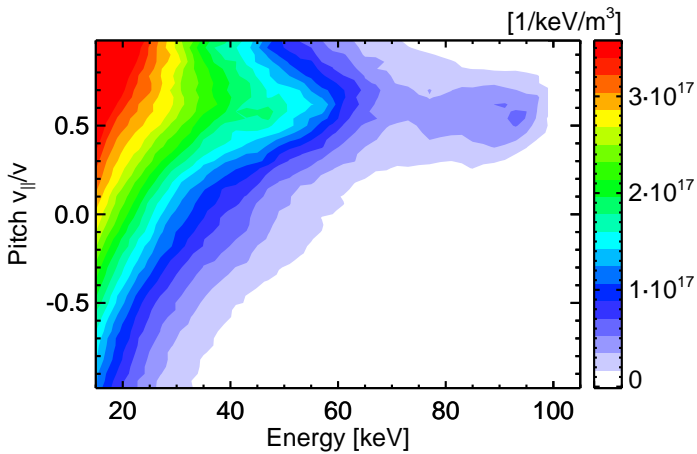

(b) $t=1.23 \mathrm{~s}$

Figure 12: Fast-ion distribution calculated by NUBEAM/TRANSP. The distribution of thermal deuterium is added according to eq. 8 .

The normalization is such, that $\int f \mathrm{~d} E \mathrm{~d} \xi=n_{\mathrm{D}}$ and $v_{\|}$and $v_{\perp}$ can be calculated straightforwardly from $E$ and $\xi$ :

$$
v_{\|}=\sqrt{\frac{2 E}{m}} \cdot \xi \quad v_{\perp}=\sqrt{\frac{2 E}{m}} \cdot \sqrt{1-\xi^{2}}
$$

The contour plots show a good agreement, overall. For a more quantitative comparison to the FIDA tomography, we have calculated energy and pitch profiles of the 2D distribution functions by integrating out the other coordinate (fig. 13). For the pitch profile, we have integrated over $E>24 \mathrm{keV}$, in order to compare only the trustworthy region of the tomographic reconstruction.

In the pitch profiles, a good agreement is found both in absolute values and shape of the profile. The main difference is that the tomography shows less pronounced peaks. This effect is also seen clearly in the contour plots for $t=0.92 \mathrm{~s}$ (figures 11 and 12). It might be a consequence of the 1st order regularization, which demands small gradients. For this particular feature of the distribution function, regularization methods, which tend to 


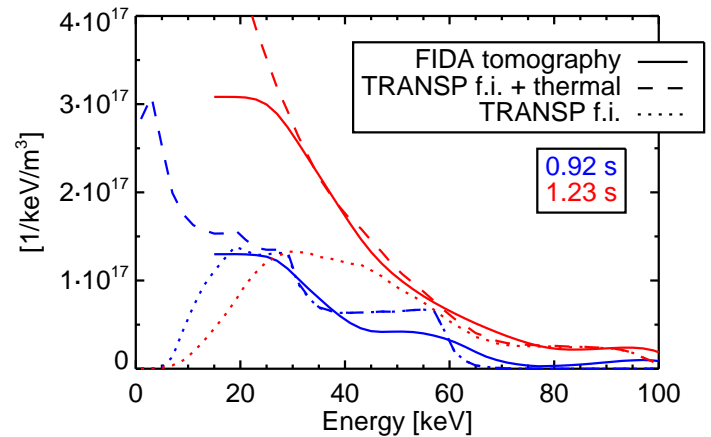

(a) $\int f(E, \xi) \mathrm{d} \xi$

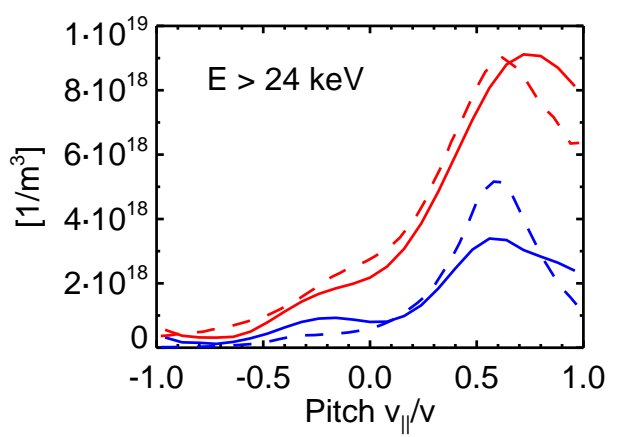

(b) $\int_{E>24 \mathrm{keV}} f(E, \xi) \mathrm{d} E$

Figure 13: Energy and pitch profiles calculated from the tomogram and the TRANSP ion distribution. $t=0.92 \mathrm{~s}$ corresponds to $60 \mathrm{keV} \mathrm{NBI}$, while $t=1.23 \mathrm{~s}$ has $93 \mathrm{keV}$ NBI in addition. In (a), the energy profile of the TRANSP fast-ion distribution (without thermal deuterium) is plotted for comparison.

pronounce peaks more strongly (e.g. Minimum Fisher information [18]), might improve the results. On the other hand, an exact reconstruction of the peaks is not so important from a physical point of view: The peaks are mainly determined by the (well-known) geometry of the NBI, while the interesting physics aspects are found elsewhere in the velocity space (e.g. high energy tails, fast-ion redistribution due to MHD etc.).

The energy profiles show also a good agreement in shape and absolute values. The strong increase of the measured fast-ion density at the second time point fits well to the TRANSP prediction. It can be seen that the thermal ions contribute a significant part to the distribution at $t=1.23 \mathrm{~s}$ due to high $T_{\mathrm{i}}$ values there and that the agreement is greatly enhanced by adding the thermal component to the TRANSP fast-ion distribution. Differences are found for $t=0.92 \mathrm{~s}$ : In the region around $50 \mathrm{keV}$ the tomography yields less fast ions, and around $65 \mathrm{keV}$ it yields more fast ions than TRANSP. This might be explainable to some extent with a too strong smoothing of the tomography. At $t=$ $1.23 \mathrm{~s}$, the extrapolative character of the tomography at energies below $\approx 24 \mathrm{keV}$ can be seen clearly, and differences to TRANSP occur. For higher energies, the tomography and TRANSP agree very well.

Overall the tomography made from the FIDA data reproduces well the fast-ion distributions of NBI at two different injection energies, and good agreement to TRANSP/NUBEAM is found. The method can therefore be applied to investigate physics mechanisms.

\section{Fast-ion redistribution by sawtooth crashes}

Sawtooth crashes can strongly redistribute fast ions, which has been demonstrated e.g. from FIDA [?] or neutron measurements [19]. The improved FIDA setup and the additional views can be used now to study the velocity dependence of this redistribution in detail.

We have analyzed discharge \#31557. The discharge was run with a magnetic field of -2.6 $\mathrm{T}$ and a plasma current of $1 \mathrm{MA}$. Fig. 14 shows time traces of the central and mid-radius 


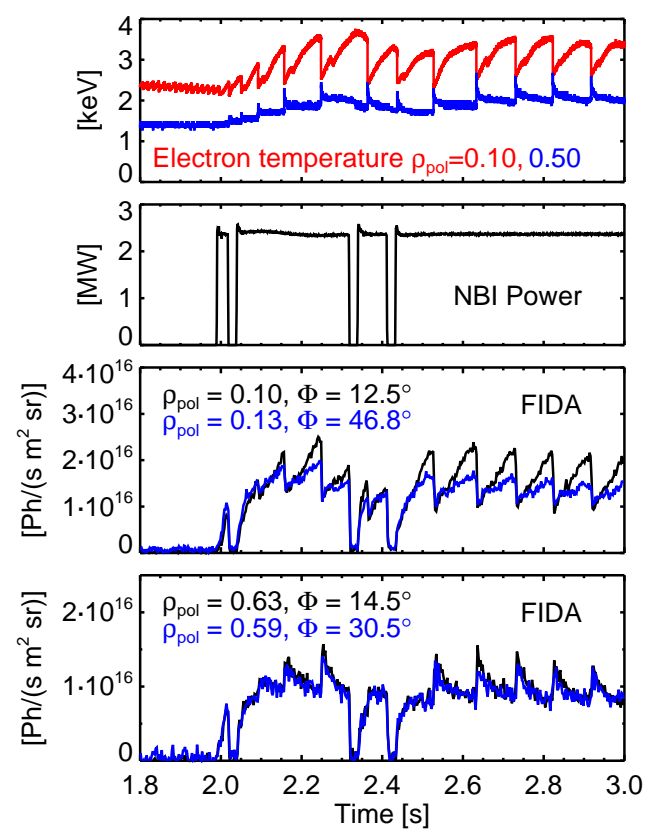

Figure 14: Representative timetraces of ASDEX Upgrade discharge \#31557. The FIDA raw signals are obtained by integrating the spectra over Doppler-shifts $\Delta \lambda=[3.7,5.0] \mathrm{nm}$

electron temperature, where strong sawtooth activity can be identified. The mid-radius time trace shows an temperature increase with each sawtooth crash, and hence lies outside of the inversion radius. The time trace of the NBI (Q3) and the FIDA raw signals for central and mid-radius LOS are shown below. The onset of NBI is followed by a rise of the FIDA radiation, which can be identified with the fast-ion density build-up. Later on, the FIDA radiation drops in the plasma center with each sawtooth crash, and rises in the outer lines of sight. This indicates a strong redistribution of the fast ions due to the crash. From the raw signals, it is already observable that the central $\Phi=10^{\circ}$ LOS, which sees mostly co-current fast ions, measures the strongest sawtooth drop, while the FIDA views, which observe more strongly gyrating fast ions have smaller sawtooth drops. Outside of the inversion, the $\Phi=10^{\circ}$ projection shows a stronger rise of FIDA radiation than a $\Phi=30^{\circ}$ projection.

This can be seen more clearly in figure 15, where the relative change ((after-before) / before) of the FIDA emission during the sawtooth crash at $2.25 \mathrm{~s}$ is shown for five FIDA views and for the two radial positions as a function of the mean observed pitch. These observations give the indication, that fast ions with high pitches $v_{\|} / v$ are more strongly expelled from the plasma center than fast ions with low pitches. This can be studied more quantitatively with the FIDA tomography method, described in section 2.6.

We have performed tomographic reconstructions before and after the sawtooth at $2.25 \mathrm{~s}$ from five FIDA views, which measure all approximately at the same radial position. The calculation was carried out for a set of LOS in the plasma center $\left(\rho_{\text {pol }} \approx 0.10\right)$ and a second set of five views outside of the sawtooth inversion $\left(\rho_{\text {pol }} \approx 0.60\right)$. The input spectra for the tomography in the plasma center are shown in fig. 16. It can be seen directly that the FIDA contribution in the spectra is significantly lower after the crash - most strongly 


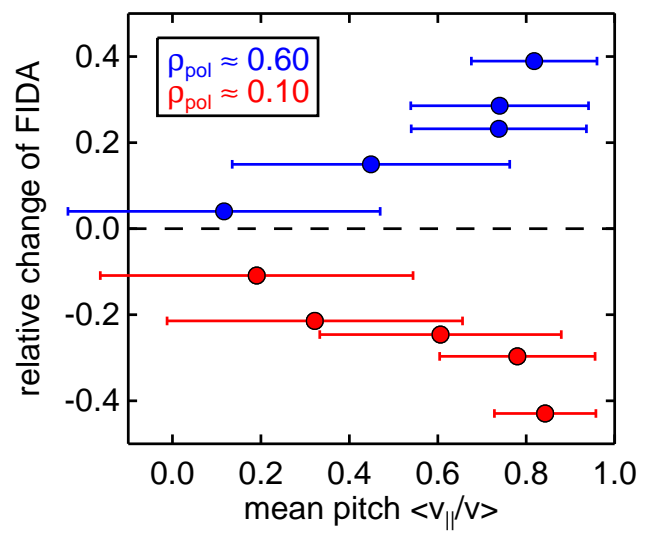

Figure 15: Relative change ((after-before) / before ) of the FIDA emission $(|\Delta \lambda|=$ $[3.5,4.5] \mathrm{nm})$ during the sawtooth crash at $2.25 \mathrm{~s}$. The $x$-axis refers to the average pitch, which is observed by the line of sight. It is calculated by integrating the weight functions over the relevant energies (20-60 keV) and wavelength range: $g(\xi)=\int_{\lambda_{1}}^{\lambda_{2}} \int_{E_{1}}^{E_{2}} W(\lambda, E, \xi) \mathrm{d} \lambda \mathrm{d} E$. The mean value of $g(\xi)$ is plotted as dot, and the standard deviation is shown with error bars. It can be seen clearly that the more tangential FIDA views have a better pitch-resolution than the perpendicular views, which can also be seen in the contour plots of the weight functions (see fig. 8).
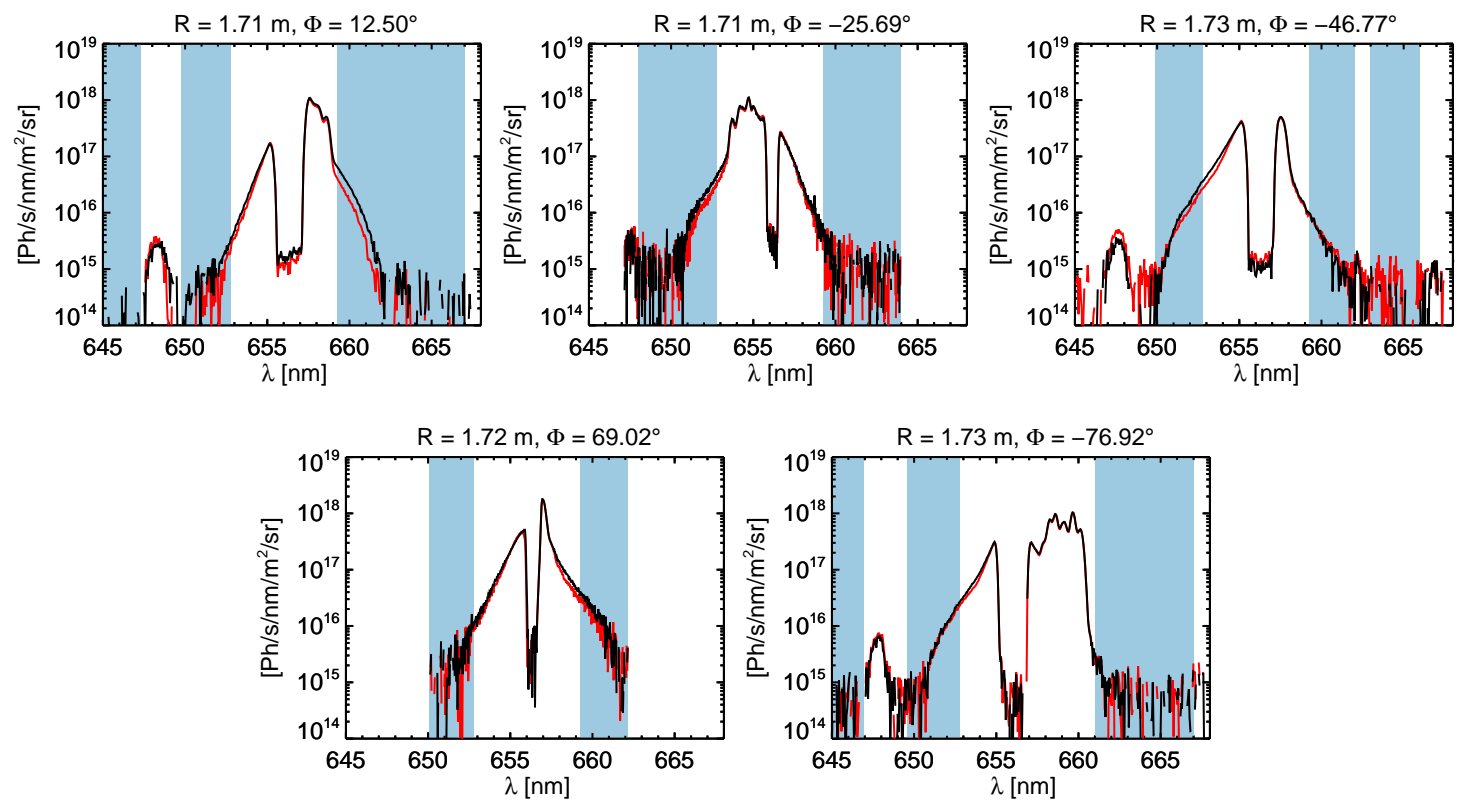

Figure 16: FIDA spectra of five views before (black) and after (red) the sawtooth crash. The tomography is calculated from the blue-shaded regions. 


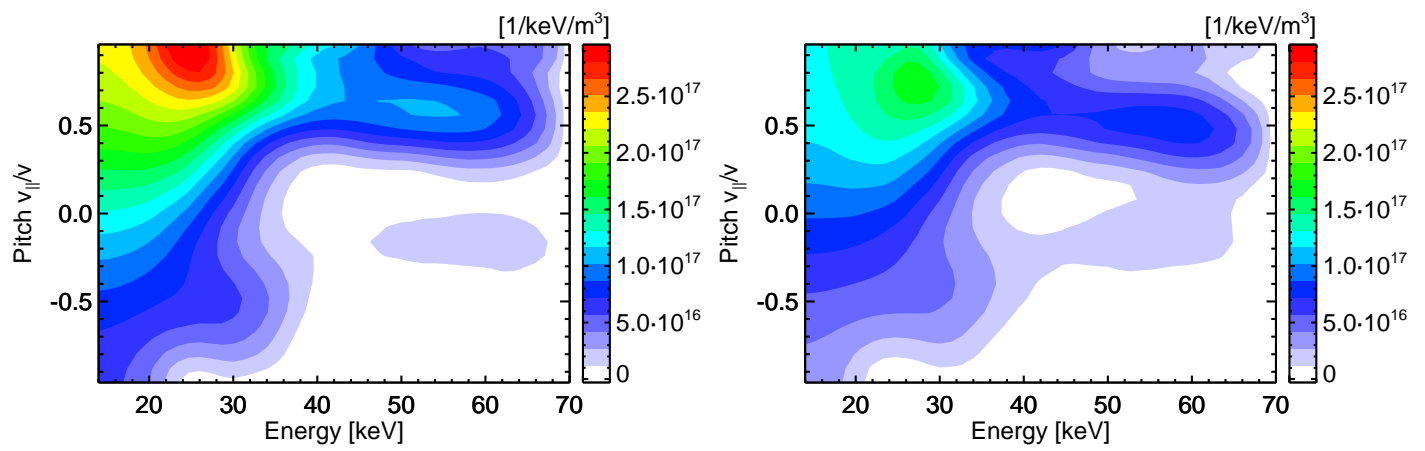

(a) Plasma center $\rho_{\text {pol }} \approx 0.10$

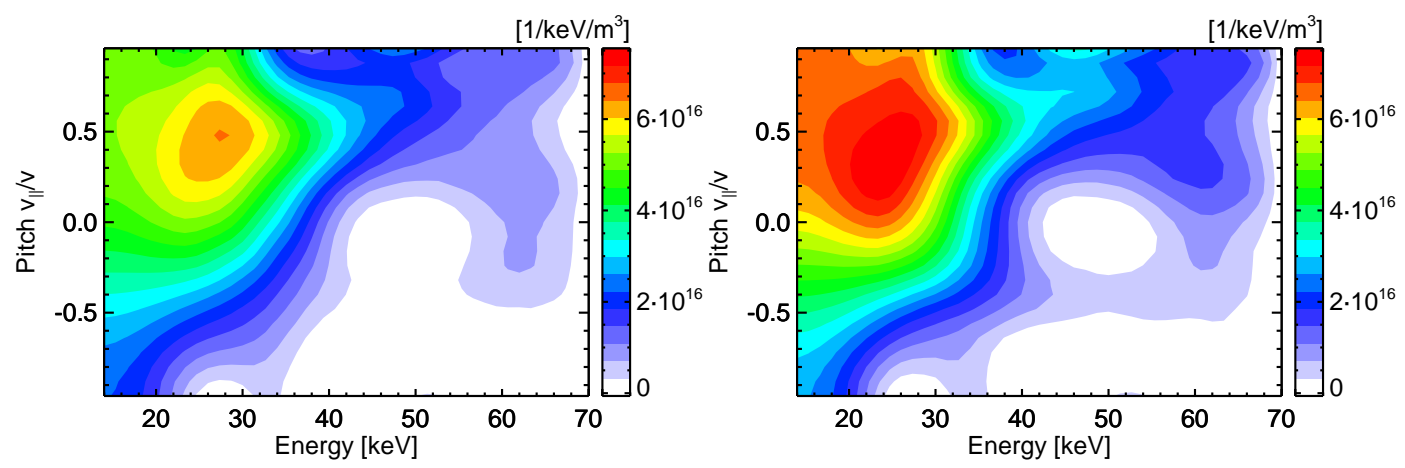

(b) $\rho_{\mathrm{pol}} \approx 0.60$

Figure 17: Tomographic reconstruction of $f\left(E, v_{\|} / v\right)$ from FIDA measurements before (left) and after a sawtooth crash

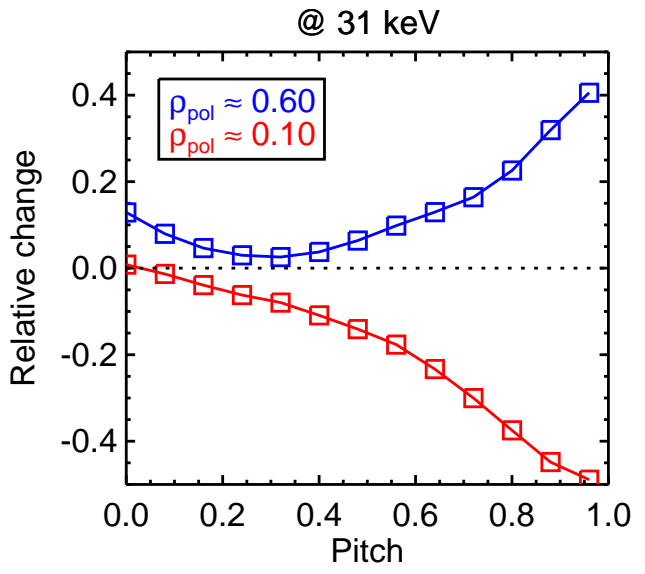

Figure 18: Pitch distribution of the relative change of $f\left(E=31 \mathrm{keV}, v_{\|} / v\right)$.

for the most tangential view $\left(\Phi=12.5^{\circ}\right)$. As in the previous section, regions with impurity lines and beam emission are excluded for the tomography, as-well as the wavelength range [-3.1 nm, $+3.1 \mathrm{~nm}]$ around the D-alpha line, which is dominated by thermal ions.

The results of the tomography are shown in fig. 17. From the reconstruction, it is possible to determine the total fast-ion density (of fast ions above $24 \mathrm{keV}$ ), and thus 
quantify the effect of the sawtooth: In the plasma center, it causes a $25 \%$ drop of total fast-ion density, while at $\rho_{\text {pol }}=0.60$ an increase of $19 \%$ is observed. With a cut of the fast-ion distribution function at constant energy, we can estimate how this total density changes are distributed along different pitches (fig. 18). It can be seen that fast ions with high pitches are much stronger expelled from the plasma core $(-50 \%)$, while more strongly gyrating fast ions with pitches close to 0 are much less affected by the sawtooth. This is in accordance with [20], where a FIDA tomography is calculated with singular value decomposition from four FIDA views in the plasma center, and a similar pitch distribution of the density change is found.

Outside of the sawtooth inversion at $\rho_{\text {pol }}=0.60$, a strong increase is found for fast ions with high pitches $(+40 \%)$, while at the same time, only little changes are observed for strongly gyrating fast ions with pitches close to 0 .

These findings of the tomography are in line with the basic trend, which is already visible in the raw data (as shown in fig. 15). However, the tomography allows a more detailed and quantitative study. For example, the influence of the changed beam attenuation due to the strongly changed kinetic profiles is taken into account, because these effects are included in the calculation of the weight functions and separate weight functions are calculated for both time points.

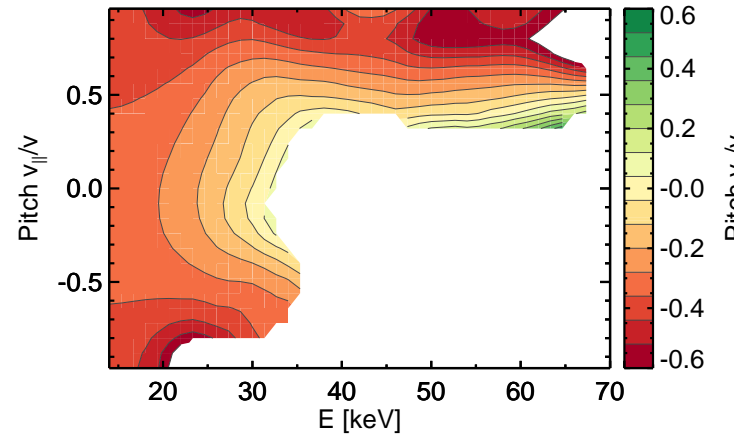

(a) $\rho_{\text {pol }}=0.10$

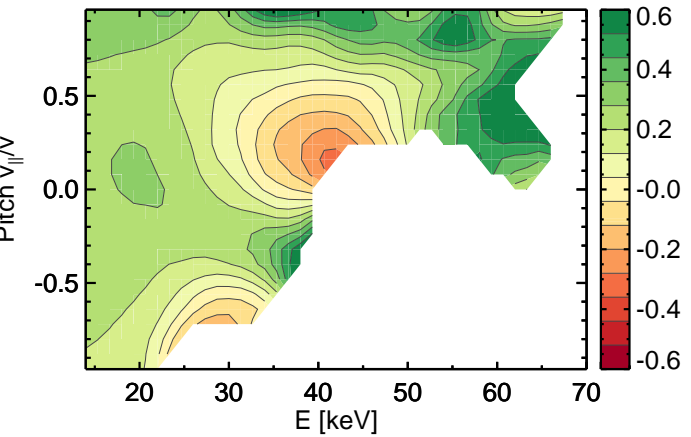

(b) $\rho_{\text {pol }}=0.60$

Figure 19: Relative change $\left(f_{\text {after }}-f_{\text {before }}\right) / f_{\text {before }}$ at two different radial positions in and outside of the sawtooth inversion. The relative difference is only well defined, if $f_{\text {before }}(E, \xi)$ is high enough. Therefore, only regions which fulfill $f_{\text {before }}(E, \xi) \geq 0.15 \cdot \max \left(f_{\text {before }}\right)$ are shown.

We can get a more detailed picture of the redistribution by calculating the relative change for the entire velocity space. This is shown for both radial positions in figure 19 . The relative difference is only well defined, if $f_{\text {before }}(E, \xi)$ is high enough. Therefore we have calculated it only for regions which fulfill $f_{\text {before }}(E, \xi) \geq 0.15 \cdot \max \left(f_{\text {before }}\right)$. It can be seen that the absolute value of the relative change is largest for pitches close to +1 for all energies, and weaker for fast ions with pitches around 0 . It is hard to make a robust statement for fast ions with very negative pitches, because there are very few fast ions due to to co-current orientation of the neutral beams. At least in the plasma center, it seems that the strength of fast-ion redistribution is more or less symmetrical with respect to $\xi=0$. For low energies, the pitch dependence of the sawtooth redistribution is rather 
weak, as it is expected for thermal particles. This pitch dependence seems to increase with energy - and a region around $\xi=0$ with very weak redistribution is seen towards higher energies. Outside of the inversion radius an overall similar shape of the relative change is found with opposite sign. This can be interpreted such that the ions from the core are redistributed further outside, keeping their energy and magnetic moment more or less constant. There are some small scale structures visible close to the $f_{\text {before }}(E, \xi) \geq$ $0.15 \cdot \max \left(f_{\text {before }}\right)$ boundary, which should be treated with care, since the region close to that boundary has larger uncertainties and the outer measurement position has less fast ions in general. The rather broad region with high redistribution around $E=60 \mathrm{keV}, \xi=$ 0.5 might be explainable by the fact that the full energy component ( $60 \mathrm{keV}$ ) of the beam is more strongly deposited in the plasma center. Hence, redistribution of those particles towards the mid-radius causes a stronger relative increase there.

In the following, we want to discuss briefly a physical interpretation of these findings. Theoretical descriptions of fast-ion sawtooth redistribution can be found in [21, 22, 23. Therein, the evolution of the helical flux $\Psi^{*}$ is modeled according to the Kadomtsev model [24] with the sawtooth crash duration $\tau_{\text {cr }}$ as free parameter. The fast temporal change $\dot{\Psi}^{*}$ of the helical flux during the crash induces an electrical field $\vec{E}$. This results in a radial $\vec{E} \times \vec{B}$-drift (with respect to the minor radius, i.e. from the plasma core to the edge or vice versa), which describes the sawtooth redistribution. This radial drift strongly depends on the helical angle $\eta=\theta-\varphi$, where $\varphi$ is the toroidal and $\theta$ the straight field line angle [25, 26] (or in good approximation for the plasma core the poloidal angle). Particles, which are closely following the field-lines (e.g. thermal particles, or fast ions with high pitches $|\xi|)$, will stay at $\eta \approx$ const and will be strongly redistributed.

In [23], it is shown that the radial $\vec{E} \times \vec{B}$-drift cancels out almost completely when averaging over all $\eta$ positions. This can be interpreted by a phase-mixing between mode and particles, which prevents particle redistribution. The combined curvature and $\nabla \vec{B}$ drift $\vec{v}_{\mathrm{D}}$ allows such a change of $\eta$ along a particle orbit:

$$
\left\langle\dot{\eta}_{\mathrm{vD}}\right\rangle \equiv \omega_{\mathrm{vD}}=\left\langle\frac{\partial \theta}{\partial R} v_{\mathrm{D}, R}-\frac{v_{\mathrm{D}, \varphi}}{R}+\frac{\partial \theta}{\partial z} v_{\mathrm{D}, z}\right\rangle \approx\left\langle\frac{\partial \theta}{\partial z} v_{\mathrm{D}, z}\right\rangle
$$

Hereby, the brackets refer to an average over one poloidal turn of the ion orbit. $v_{\mathrm{D}, R}, v_{\mathrm{D}, \varphi}$ and $v_{\mathrm{D}, z}$ are the $R, \varphi$ and $z$ components of $\vec{v}_{\mathrm{D}}$, whereby the first two can be neglected in good approximation compared to the $z$ component. A particle can escape the sawtooth redistribution, if $\left|\omega_{\mathrm{vD}}\right|$ is high enough with respect to the angular frequency $\omega_{\mathrm{cr}}=\pi / \tau_{\mathrm{cr}}$ associated with the sawtooth crash duration $\tau_{\text {cr }}$ (assuming that half a turn is enough to detach particles [23]). The latter can be estimated from core soft-x-ray signals at $\tau_{\text {cr }} \approx 80 \mu \mathrm{s}$ (see fig. 20a). We have calculated the ratio $\left|\omega_{\mathrm{vD}}\right| / \omega_{\mathrm{cr}}$ with an orbit following code, using a CLISTE [27] magnetic equilibrium reconstruction, for the tomography measurement position in the plasma center. It is shown in fig. 20 b as a function of energy and pitch, along with a classification of the orbits.

It can be seen that $\left|\omega_{\mathrm{vD}}\right| / \omega_{\mathrm{cr}} \ll 1$ for strongly passing ions with high $|\xi|$, which is in accordance with the strong redistribution measured at high pitches. In contrast, a broad region around $\xi=0$ can be seen with $\left|\omega_{\mathrm{vD}}\right| / \omega_{\mathrm{cr}}>1$ and hence a weak sawtooth redistribution. This is in qualitative agreement to the tomography results. Furthermore, 

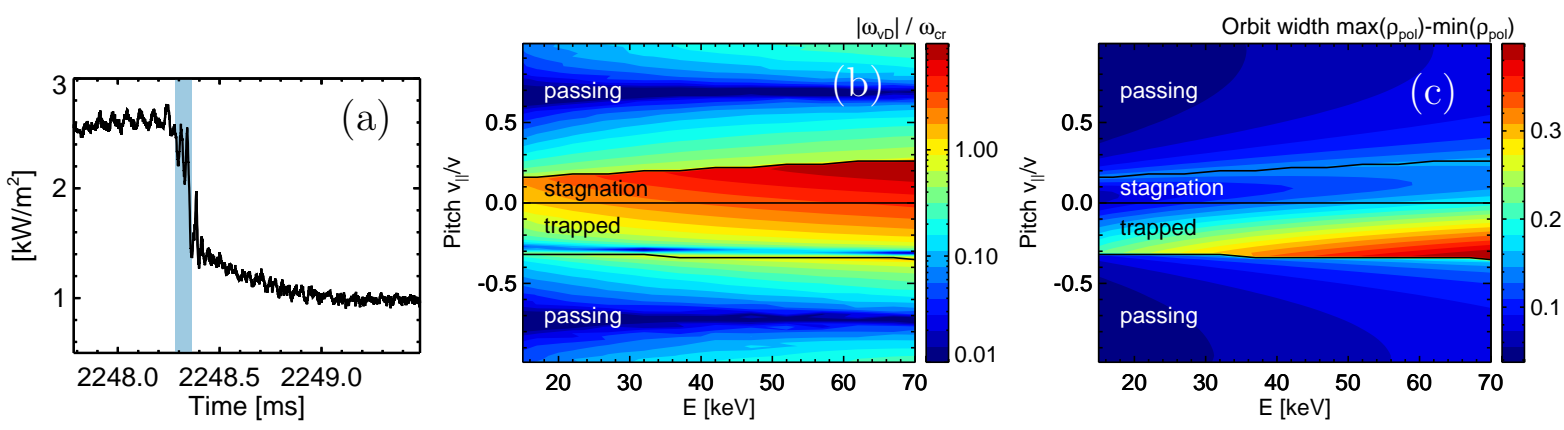

Figure 20: (a) Average of two central SXR LOS signals with similar poloidal angle but at different toroidal angle. By the averaging, the signature of the $(1,1)$ mode is reduced and the evolution of the sawtooth itself can be seen more clearly. From the time trace, we can conclude that the sawtooth crash lasts $\tau_{\text {cr }} \approx 80 \mu$ s (blue-shaded area). The latter is in good agreement with the prediction from [28] for collisionless reconnection dynamics. (b) Ratio of helical precession $\left|\omega_{\mathrm{vD}}\right|$ (calculated for the central position of the tomography) and angular crash frequency $\omega_{\mathrm{cr}}$. In the limit of $|q-1| \ll 1,\left|\omega_{\mathrm{vD}}\right| / \omega_{\mathrm{cr}} \gtrsim 1$ is a criterion that fast ions may escape the reconnection ( $q$ being the safety factor). (c) Width of the fast-ion orbits in terms of $\rho_{\text {pol }}\left(\max \left(\rho_{\text {pol }}\right)-\min \left(\rho_{\text {pol }}\right)\right.$ along the guiding-center orbit $)$, for the same position as in (b).

it can be seen that $\left|\omega_{\mathrm{vD}}\right| / \omega_{\text {cr }}$ increases strongly with the energy (logarithmic scale in fig. $20 \mathrm{p}$ ), which can explain why we measure lower relative changes around $\xi \approx 0$ with increasing energy.

The velocity space region with weak sawtooth redistribution is mostly filled with trapped ions for $\xi<0$ and with passing, but not axis-encircling, ions for $\xi>0$ (i.e. stagnation orbits). For passing ions, this simple model predicts a much lower $\omega_{\mathrm{vD}}$. This can be understood directly from equation (10): While the drift $v_{\mathrm{D}, z}$ remains more or less constant during an orbit, and points always downwards, $\frac{\partial \theta}{\partial z}$ (i.e. the field-line slope w.r.t. $z$ ) has a different sign on the high field side than on the low field side (LFS). Passing particles spend approximately equal time on both sides, and hence $\omega_{\mathrm{vD}}$ is low. In contrast, deeply trapped particles spend more time during their orbit on the LFS, and stagnation orbits stay completely on the LFS - leading to much higher $\omega_{\mathrm{vD}}$.

Another effect, which can lead to weaker sawtooth redistribution are large orbit widths. However, as fig. 20k shows, all orbits with $\xi \in[0 ; 1]$ have comparable widths and hence cannot explain the measured variation of the redistribution in that interval. Only the trapped particles have significantly larger orbit widths, but they are still much smaller than the sawtooth mixing radius. Hence we can conclude that the orbit widths do not play an important role in this particular case. It has to be noted that these simple considerations can only provide a basic picture of the underlying physics. For example, the influence of the helical $(1,1)$ mode on the particle orbits [29] is neglected here. Nevertheless, a qualitative explanation, why particles with low $|\xi|$ are less affected by the sawtooth can be given, while the exact shape (or the absolut values) of the measured relative change cannot be determined from this basic considerations. For that, more sophisticated modelling is needed (e.g. as described in [21, 22, 23]), and the results of the FIDA tomography could allow to verify these models. 


\section{Conclusion and outlook}

The FIDA diagnostic at ASDEX Upgrade has been upgraded from two viewing arrays and a single third view line of sight towards five full viewing arrays. Each view consists of several radially distributed lines of sight and has a different angle towards the magnetic field $\Phi$. The latter determines the observed region in velocity space, and hence a fairly uniform distribution of angles (in the plasma center) has been chosen: $\Phi \approx 10^{\circ},-20^{\circ},-50^{\circ}, 70^{\circ}$ and $85^{\circ}$. The FIDA spectrometer has been equipped with a wire to block the central D-alpha line as replacement for a previously used high-pass interference filter. This allows simultaneous measurement of the red and blue part of the Doppler spectrum and hence doubles the observable information about the velocity space. Furthermore, the spectrometer capacities have been enhanced from 16 LOS channels to 37 channels, such that 37 lines of sights can be measured at the same time.

With these new diagnostic capabilities, it is possible to reconstruct the fast-ion velocity distribution function from the Doppler spectra by tomography under a wide range of plasma parameters. Hence, velocity-space resolved fast-ion transport studies are possible. The accuracy of the tomography is studied in a comparison of $60 \mathrm{keV}$ and $93 \mathrm{keV}$ NBI phases. The different energies are clearly resolved by the tomography. The fast-ion pitch distribution is well in agreement with the geometrical setup of the NBI, and a good agreement to theoretical predictions by NUBEAM/TRANSP is found for both cases.

As a physical application, fast-ion redistribution in the presence of sawtooth crashes is studied. A $25 \%$ overall drop of fast-ion density is observed in the plasma center in line with a $19 \%$ increase at $\rho_{\text {pol }}=0.6$. For both positions, the strongest redistribution is seen for fast ions with large pitches close to 1 . The tomographic reconstruction allowed us to estimate the fast-ion redistribution quantitatively and energy and pitch resolved. The basic trends are, however, already visible in the raw data, by comparing signals from different views.

Within this work, we have neglected the spatial extension of the FIDA measurement volumes during the tomography process. Instead, we have combined five views, and assumed that they measure at the same spatial position. The reasonable results and good agreement to TRANSP justify these assumptions. However, an improvement would be possible by extending the tomography with a radial coordinate (e.g. $\left.\rho_{\text {pol }}\right)$. Consequently, the weight functions $W$ would become functions of $\rho_{\text {pol }}$, and the tomography would yield a $3 \mathrm{D}$ distribution function $f\left(E, \xi, \rho_{\mathrm{pol}}\right)$. While this increases the complexity and calculation time of the tomography strongly, it would allow to directly derive important profiles from the FIDA measurements, such as the fast-ion density, fast-ion pressure or fast-ion current (NBCD) profiles.

\section{Acknowledgement}

This work has been carried out within the framework of the EUROfusion Consortium and has received funding from the Euratom research and training programme 2014-2018 under grant agreement No 633053. The views and opinions expressed herein do not necessarily reflect those of the European Commission. 


\section{References}

[1] P. A. Schneider, H. Blank, B. Geiger, K. Mank, S. Martinov, F. Ryter, M. Weiland, A. Weller, and the ASDEX Upgrade Team. A new compact solid-state neutral particle analyser at ASDEX Upgrade: Setup and physics modeling. Review of Scientific Instruments, 86(7):-, 2015.

[2] S. K. Nielsen, M. Stejner, J. Rasmussen, A. S. Jacobsen, S. B. Korsholm, F. Leipold, M. Maraschek, F. Meo, P. K. Michelsen, D. Moseev, M. Salewski, M. Schubert, J. Stober, W. Suttrop, G. Tardini, and D. Wagner. Measurements of the fast-ion distribution function at ASDEX upgrade by collective Thomson scattering (CTS) using active and passive views. Plasma Physics and Controlled Fusion, 57(3):035009, 2015 .

[3] G. Tardini, A. Zimbal, B. Esposito, F. Gagnon-Moisan, D. Marocco, R. Neu, H. Schuhmacher, and the ASDEX Upgrade Team. First neutron spectrometry measurements in the ASDEX Upgrade tokamak. Journal of Instrumentation, 7(03):C03004, 2012.

[4] A. Jacobsen, M. Salewski, J. Eriksson, G. Ericsson, S. Korsholm, F. Leipold, S. Nielsen, J. Rasmussen, M. Stejner, and J. E. Contributors. Velocity-space sensitivity of neutron spectrometry measurements. Nuclear Fusion, 55(5):053013, 2015.

[5] W. Heidbrink, K. Burrell, Y. Luo, N. Pablant, and E. Ruskov. Hydrogenic fast-ion diagnostic using Balmer-alpha light. Plasma physics and controlled fusion, 46(12):1855, 2004 .

[6] W. Heidbrink, D. Liu, Y. Luo, E. Ruskov, and B. Geiger. A code that simulates fastion D-alpha and neutral particle measurements. Comm. Comp. Physics, 8, 2010.

[7] M. Salewski, B. Geiger, S. Nielsen, H. Bindslev, M. García-Muñoz, W. Heidbrink, S. Korsholm, F. Leipold, F. Meo, P. Michelsen, D. Moseev, M. Stejner, G. Tardini, and the ASDEX Upgrade team. Tomography of fast-ion velocity-space distributions from synthetic CTS and FIDA measurements. Nuclear Fusion, 52(10):103008, 2012.

[8] M. Salewski, B. Geiger, A. Jacobsen, M. García-Muñoz, W. Heidbrink, S. Korsholm, F. Leipold, J. Madsen, D. Moseev, S. Nielsen, J. Rasmussen, M. Stejner, G. Tardini, M. Weiland, and the ASDEX Upgrade Team. Measurement of a 2D fast-ion velocity distribution function by tomographic inversion of fast-ion D-alpha spectra. Nuclear Fusion, 54(2):023005, 2014.

[9] B. Geiger, M. Weiland, A. Mlynek, M. Reich, A. Bock, M. Dunne, R. Dux, E. Fable, R. Fischer, M. Garcia-Munoz, J. Hobirk, C. Hopf, S. Nielsen, T. Odstrcil, C. Rapson, D. Rittich, F. Ryter, M. Salewski, P. A. Schneider, G. Tardini, and M. Willensdorfer. Quantification of the impact of large and small-scale instabilities on the fast-ion confinement in ASDEX Upgrade. Plasma Physics and Controlled Fusion, 57(1):014018, 2015. 
[10] B. Geiger, R. Dux, R. McDermott, S. Potzel, M. Reich, F. Ryter, M. Weiland, D. Wünderlich, ASDEX Upgrade team, and M. Garcia-Munoz. Multi-view fast-ion D-alpha spectroscopy diagnostic at ASDEX Upgrade. Review of Scientific Instruments, 84(11):113502, 2013.

[11] Y. Luo, W. W. Heidbrink, K. H. Burrell, D. H. Kaplan, and P. Gohil. Measurement of the $\mathrm{D}_{\alpha}$ spectrum produced by fast ions in DIII-D. Review of Scientific Instruments, 78(3):-, 2007.

[12] M. Podestà, W. W. Heidbrink, R. E. Bell, and R. Feder. The NSTX fast-ion D-alpha diagnostic. Review of Scientific Instruments, 79(10), 2008.

[13] M. Salewski, B. Geiger, D. Moseev, W. W. Heidbrink, A. S. Jacobsen, S. B. Korsholm, F. Leipold, J. Madsen, S. K. Nielsen, J. Rasmussen, M. Stejner, M. Weiland, and the ASDEX Upgrade Team. On velocity-space sensitivity of fast-ion D-alpha spectroscopy. Plasma Physics and Controlled Fusion, 56(10):105005, 2014.

[14] R. Hawryluk and et al. An Empirical Approach to Tokamak Transport. in Physics of Plasmas Close to Thermonuclear Conditions, 1:19-46, 1980.

[15] A. Pankin, D. McCune, R. Andre, G. Bateman, and A. Kritz. The tokamak Monte Carlo fast ion module NUBEAM in the National Transport Code Collaboration library. Computer Physics Communications, 159(3):157 - 184, 2004.

[16] M. Salewski, B. Geiger, W. W. Heidbrink, A. S. Jacobsen, S. B. Korsholm, F. Leipold, J. Madsen, D. Moseev, S. K. Nielsen, J. Rasmussen, L. Stagner, D. Steeghs, M. Stejner, G. Tardini, and M. Weiland. Doppler tomography in fusion plasmas and astrophysics. Plasma Physics and Controlled Fusion, 57(1):014021, 2015.

[17] A. S. Jacobsen, L. Stagner, M. Salewski, B. Geiger, W. W. Heidbrink, S. B. Korsholm, F. Leipold, S. K. Nielsen, J. Rasmussen, M. Stejner, H. Thomsen, M. Weiland, and the ASDEX Upgrade team. Inversion methods for fast-ion velocity-space tomography in fusion plasmas. Plasma Physics and Controlled Fusion, (submitted), 2015.

[18] M. Anton, H. Weisen, M. J. Dutch, W. von der Linden, F. Buhlmann, R. Chavan, B. Marletaz, P. Marmillod, and P. Paris. X-ray tomography on the TCV tokamak. Plasma Physics and Controlled Fusion, 38(11):1849, 1996.

[19] J. A. Lovberg, W. W. Heidbrink, J. D. Strachan, and V. S. Zaveryaev. Neutron sawtooth behavior in the PLT, DIII-D, and TFTR tokamaks. Physics of Fluids B: Plasma Physics, 1(4):874-892, 1989.

[20] B. Geiger, M. Weiland, A. Jacobsen, D. Rittich, R. Dux, R. Fischer, C. Hopf, M. Maraschek, R. McDermott, S. Nielsen, T. Odstrcil, M. Reich, F. Ryter, M. Salewski, P. Schneider, and G. Tardini. Fast-ion transport and neutral beam current drive in ASDEX upgrade. Nuclear Fusion, 55(8):083001, 2015.

[21] Y. Kolesnichenko and Y. Yakovenko. Theory of fast ion transport during sawtooth crashes in tokamaks. Nuclear Fusion, 36(2):159, 1996. 
[22] Y. I. Kolesnichenko, V. V. Lutsenko, Y. V. Yakovenko, and G. Kamelander. Theory of fast ion transport induced by sawtooth oscillations: Overview and new results. Physics of Plasmas, 4(7), 1997.

[23] F. Jaulmes, E. Westerhof, and H. de Blank. Redistribution of fast ions during sawtooth reconnection. Nuclear Fusion, 54(10):104013, 2014.

[24] B. B. Kadomtsev. On disruptive instability in tokamaks. Soviet Journal of Plasma Physics, 1:710-715, October 1975.

[25] J. W. Connor and R. J. Hastie. The effect of shaped plasma cross sections on the ideal internal kink mode in a tokamak. Technical Report CLM-M106, Culham Laboratory, 1985 .

[26] M. Schittenhelm and H. Zohm. Analysis of coupled MHD modes with Mirnov probes in ASDEX Upgrade. Nuclear Fusion, 37(9):1255, 1997.

[27] P.J. McCarthy, P. Martin, W. Schneider. The CLISTE interpretive equilibrium code. IPP Report 5/85, Max-Planck-Institut für Plasmaphysik, Garching, Germany, 1999.

[28] L. Zakharov, B. Rogers, and S. Migliuolo. The theory of the early nonlinear stage of $\mathrm{m}=1$ reconnection in tokamaks. Physics of Fluids B, 5(7), 1993.

[29] D. Pfefferlé, J. Graves, W. Cooper, C. Misev, I. Chapman, M. Turnyanskiy, and S. Sangaroon. NBI fast ion confinement in the helical core of MAST hybrid-like plasmas. Nuclear Fusion, 54(6):064020, 2014. 